\title{
Erratum: Relaxion fluctuations (self-stopping relaxion) and overview of relaxion stopping mechanisms
}

\author{
Nayara Fonseca, ${ }^{a, b}$ Enrico Morgante, ${ }^{a, c}$ Ryosuke Sato ${ }^{a}$ and Géraldine Servant ${ }^{a, d}$ \\ ${ }^{a} D E S Y$, \\ Notkestrasse 85, D-22607 Hamburg, Germany \\ ${ }^{b}$ Abdus Salam International Centre for Theoretical Physics, \\ Strada Costiera 11, 34151, Trieste, Italy \\ ${ }^{c}$ PRISMA ${ }^{+}$Cluster of Excellence and Mainz Institute for Theoretical Physics, \\ Johannes Gutenberg-Universität Mainz, D-55099 Mainz, Germany \\ ${ }^{d}$ II. Institute of Theoretical Physics, University of Hamburg, \\ D-22761 Hamburg, Germany \\ E-mail: nfonseca@ictp.it, emorgant@uni-mainz.de, ryosuke.sato@desy.de, \\ geraldine.servant@desy.de
}

ERRATUM TO: JHEP05(2020)080

ABSTRACT: We correct a minor point in section 3.2 of "Relaxion Fluctuations (Self-stopping Relaxion) and Overview of Relaxion Stopping Mechanisms", JHEP 05 (2020) 080. Furthermore, we clarify the origin of equation (4.25). Finally, we add some plots that are of interest.

ArXiv EPRINT: 1911.08473 


\section{Contents}

1 Relaxation without inflation: self-stopping relaxion $\quad 1$

2 Relaxation with vector boson production 1

$\begin{array}{llr}3 & \text { New plots } & 1\end{array}$

\section{Relaxation without inflation: self-stopping relaxion}

In the scenario in which the relaxation stage takes place after inflation, three scenarios are considered, corresponding to benchmark points labelled as $\mathbf{e}, \mathbf{f}, \mathbf{g}$. In the original version of the paper, it was stated on page 22 that the relaxion-Higgs mixing makes the relaxion efficiently decay before BBN in scenarios (e) and (f), but not in $(\mathbf{g})$. This statement is not correct, as the relaxion decays efficiently in all three presented benchmarks. This is not true in the entire parameter space presented in figure 5 of the paper, though. Indeed, the lifetime can be larger than $1-100 \mathrm{sec}$ in a narrow region with small coupling $g^{\prime}$ and cut-off $\Lambda$. In this region, the relaxion can be made unstable by adding the term in eq. (3.35). The discussion below eq. (3.35) is still valid, as a coupling of the relaxion to photons can always be added to the model, and its consequences are analysed here.

\section{Relaxation with vector boson production}

We also want to clarify a small point concerning eq. (4.25). Physically, this condition is equivalent to eq. (3.7), as it ensures the adiabaticity of the Higgs evolution. While in eq. (3.7) we imposed the condition on the Higgs VEV $v$, in (4.25) we imposed it on the quadratic term in the Lagrangian $\mu_{h}$, to facilitate the comparison with the results of ref. [4]. The difference between the resulting equations is a factor $\lambda^{1 / 2} \approx 0.36$, which has no visible effect on the results.

\section{New plots}

Finally, we would like to add some plots that help in clarifying the values of different quantities. Figure 1 of this erratum complements figure 4 of the original paper, with a projection of the parameter space onto the plane $\Lambda_{b}, M_{I} / \Lambda$. Figure 2 complements figure 3 of the original paper with the contours of the ratio of the minimal number of efolds in the "GKR" and "fragmentation" scenarios, highlighting the lower value of $N_{e}$ required in the fragmentation case. Finally, figure 3 refers to the scenario with EW gauge boson production during inflation described in section 4.2, and highlights the contours for the minimal and maximal value of the Hubble rate $H_{I}$ (in $\mathrm{GeV}$ ) and of the number of efolds of inflation that relaxation takes to complete. 


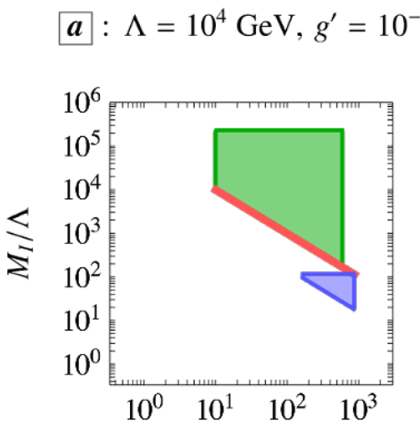

$\Lambda_{b}[\mathrm{GeV}]$

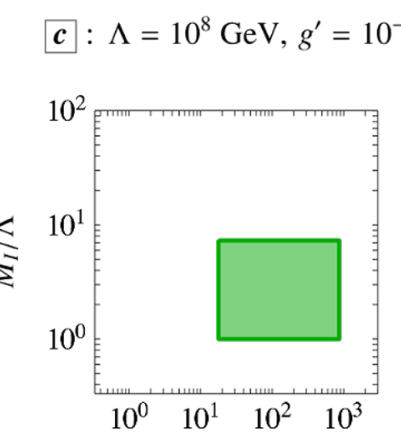

$\Lambda_{b}[\mathrm{GeV}]$

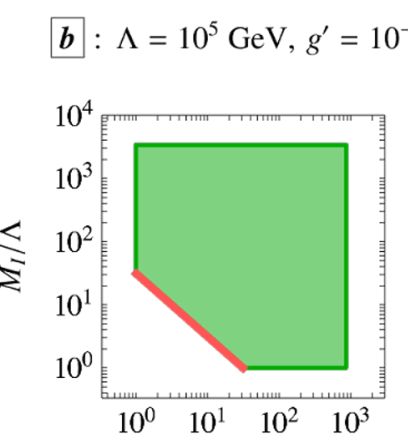

$\Lambda_{b}[\mathrm{GeV}]$

d $: \Lambda=10^{5} \mathrm{GeV}, g^{\prime}=10^{-35}$

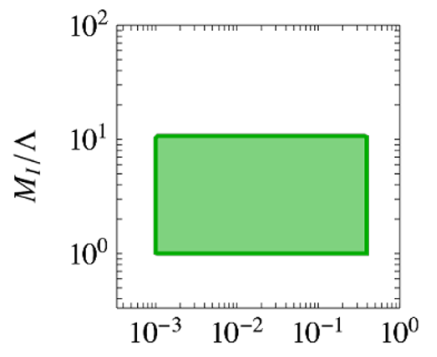

$\Lambda_{b}[\mathrm{GeV}]$

Figure 1. Same as figure 4 of the paper. Parameter space of the relaxion mechanism taking place with Higgs-dependent barriers, during inflation, where the relaxion is a subdominant component of the energy density of the universe (non-QCD case of ref. [1]), as described in section 3.1 of the paper. In blue the 'fragmentation' scenario, in red 'large barriers' and in green 'GKR'. For each benchmark point defined in section 3.1, we show the corresponding distinct regions in the plane $\Lambda_{b}$, $M_{I} / \Lambda$, in addition to the plots shown in figure 4 of the paper. In the Fragmentation case, the scale of inflation $M_{I}$ is closer to the cut-off $\Lambda$ than in the other scenarios.

Open Access. This article is distributed under the terms of the Creative Commons Attribution License (CC-BY 4.0), which permits any use, distribution and reproduction in any medium, provided the original author(s) and source are credited. 


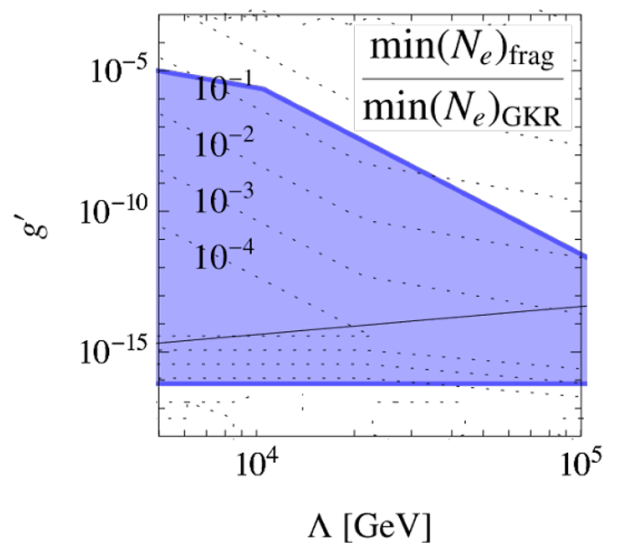

Figure 2. Same as in figure 3 of the paper. In the scenario where relaxation takes place during an inflation era, ratio of the minimal number of efolds in the "GKR" and "fragmentation" scenarios discussed in section 3.1. The coloured region corresponds to the region where fragmentation can stop the relaxion as an alternative mechanism to Hubble friction like in the GKR scenario. This plot shows that a smaller number if efolds is required to stop with fragmentation. Below the solid line the field excursion $\Lambda / g^{\prime}$ is super-Planckian. 

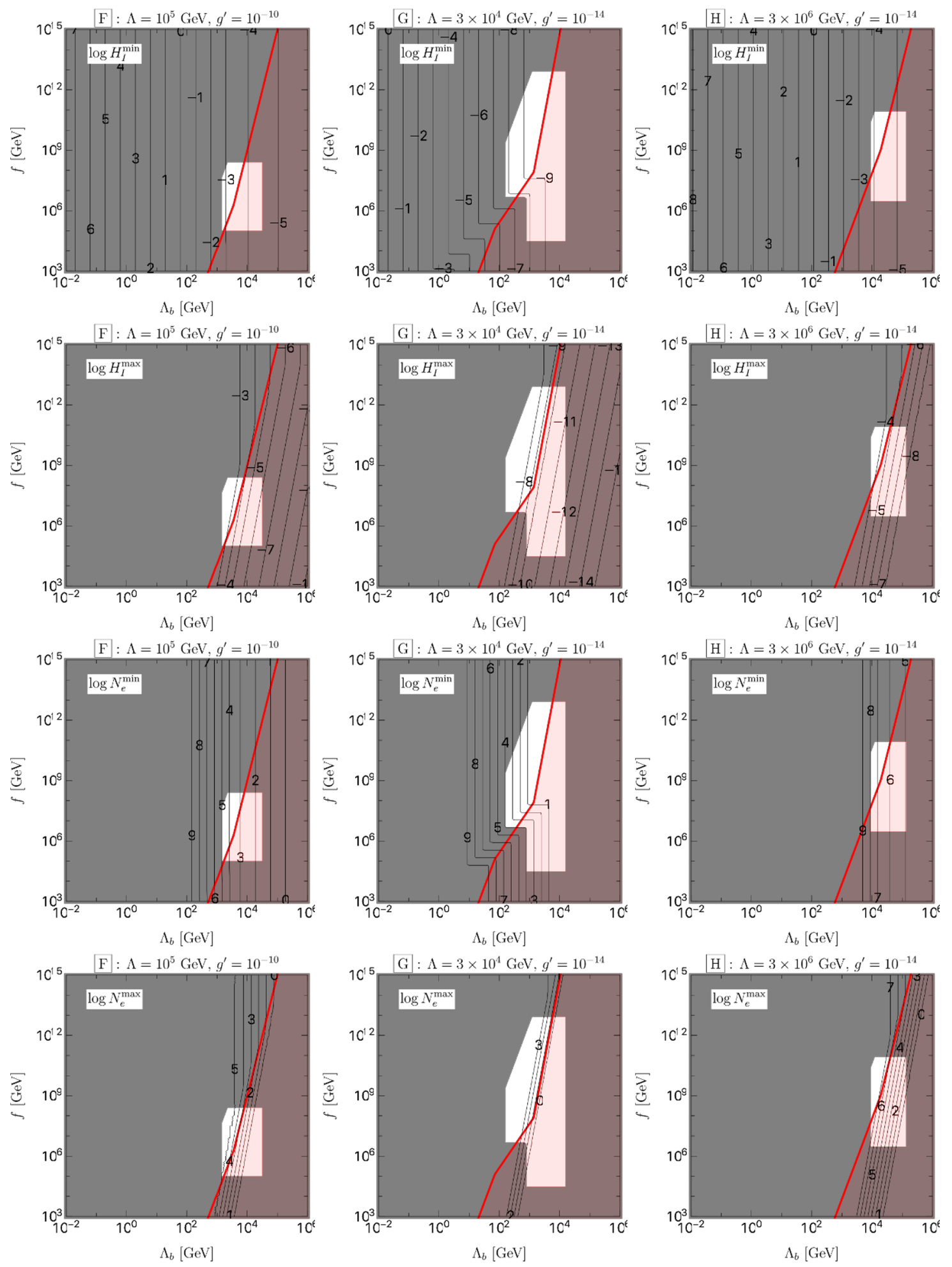

Figure 3. Same as in figure 34 of the paper. In the scenario with EW gauge boson production during inflation described in section 4.2, we highlight the contours for the minimal and maximal value of the Hubble rate $H_{I}$ (in $\mathrm{GeV}$ ) and of the number of efolds of inflation that relaxation takes to complete, for each benchmark point $F, G, H$. 